\title{
Traumatic Pseudoaneurysm of the Superficial Palmar Arch: A Case Report and Review of the Literature
}

\author{
Nikolaos Schoretsanitis $^{1} \cdot$ Erchan Moustafa $^{2} \cdot$ Efthimios Beropoulis $^{1} \cdot$ Chris Argyriou $^{1}$. \\ George S. Georgiadis ${ }^{1}$ - Efstratios Georgakarakos ${ }^{1}$
}

Received: 27 January 2015 / Accepted: 27 April 2015 /Published online: 6 May 2015

(C) Society of the Hand \& Microsurgeons of India 2015

Keywords Hand · Pseudoaneurysm · Superficial palmar arch $\cdot$ Surgical resection $\cdot$ Ultrasound

\section{Introduction}

Pseudoaneurysms of the superficial palmar arch are rare entities and only a few cases have been described in the English literature [1-7]. The majority of them develop after lesion of the artery due to penetrating trauma, previous surgery and arterial puncture. Diagnosis depends on high clinical suspicion of the disease, especially in the presence of a pulsatile mass. Below we present a case of a false aneurysm of the superficial palmar arch in a 27 year-old woman following injury with a knife that was treated surgically in our department.

\section{Case Presentation}

A 27 year old-woman suffered an accidental laceration in her palm with a kitchen knife. She was initially treated in the emergency department and discharged home with instructions for conservative treatment. The woman subsequently devel-

Efstratios Georgakarakos efstratiosgeorg@gmail.com

1 Department of Vascular Surgery, "Democritus" University of Thrace, University Hospital of Alexandroupolis, 68100 Dragana, Alexandroupolis, Greece

2 2nd Department of Surgery, "Democritus" University of Thrace, Medical School, University Hospital of Alexandroupolis, Alexandroupolis, Greece oped a swollen, pulsatile, painful and tender mass to her palm at the site of the injury (Fig. 1). Three weeks after the laceration she consulted an orthopedic surgeon and she was accordingly referred to our department for further management. Physical examination revealed adequate perfusion to the fingers with no functional or sensory impairment. A duplex ultrasound was performed, revealing a $2 \times 2.5 \mathrm{~cm}$ pulsatile mass containing no thrombotic material (Fig. 2a and b). The woman was diagnosed with a pseudoaneurysm of the superficial palmar arch and surgical repair was scheduled.

Surgery was performed under local anesthesia. Our practice is to apply a tourniquet control brachially without inflation. The inflation is performed only in cases of intraoperative hemorrhage that cannot be effectively controlled with manual compression. The mass was dissected from the surrounding tissue and ligated at its base (Fig. 3a-c). No anticoagulation was administrated during or after surgery. We did not perform a histologic examination as it was evident from the nature of injury that it was a false aneurysm. The patient was discharged home the same day. After 6 months of follow-up based on clinical assessment exclusively, the patient was free of complaints, the wound healed perfectly with intact hand function, normal movement and sensation and no numbness.

\section{Discussion}

Pseudoaneurysms or false aneurysms of the palmar arch are rare entities with only few cases described in the literature. False aneurysms differ from true aneurysms with respect to the distortion of arterial architecture. True aneurysms involve a dilatation of all three layers of the arterial wall, whereas in a false aneurysm the architecture of the vessel wall is not respected. There is a disruption of the vessel wall of the artery and a leakage of arterial blood from the artery into the 


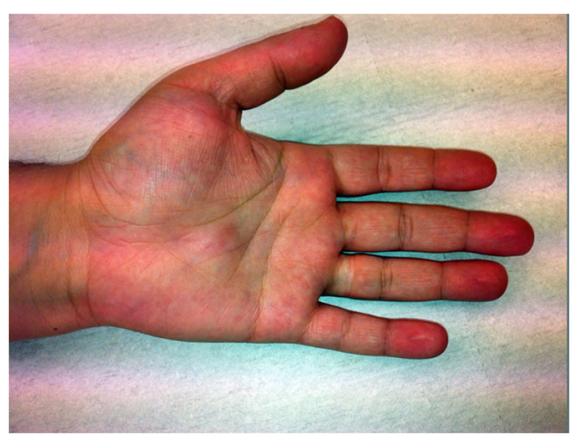

Fig. 1 Preoperative appearance with a pulsatile mass in the middle of the palm in a 27 -year-old woman

surrounding tissue with a communication between the originating artery and the resultant adjacent cavity. Their clinical presentation includes pain, swelling and a pulsatile mass in the palm near the site of the injury. They can be complicated by infection, formation of abscess, arterial occlusion and nerve compression [7].

Due to the rarity of these lesions the differential diagnosis can be quite challenging. Lipomas, incision cysts and fibromas have to be excluded in order to make the correct diagnosis. In a post traumatic scenario, the retention of a foreign body and the formation of an abscess should be taken into consideration, but the pulsatility of the mass should raise suspicion to the physician over the presence of a false aneurysm [6]. A concern should be also raised when a pulsatile mass arises progressively [3]. Therefore, follow-up is essential after a
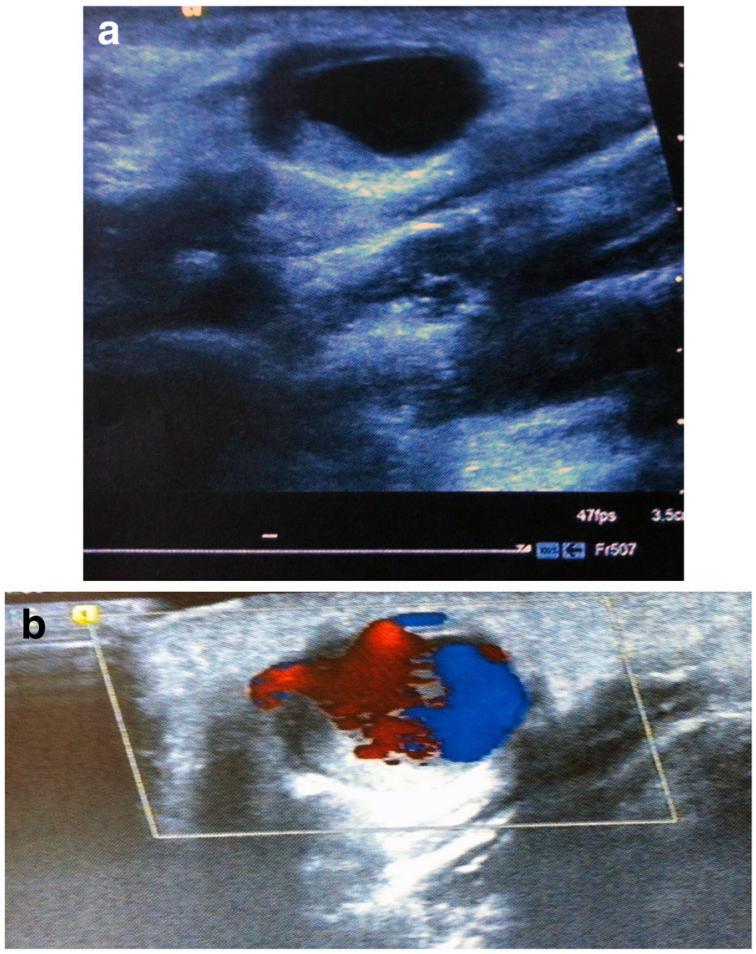

Fig. 2 a. Duplex ultrasound of the pseudoaneurysm. b. Sonographic image with color Doppler flow
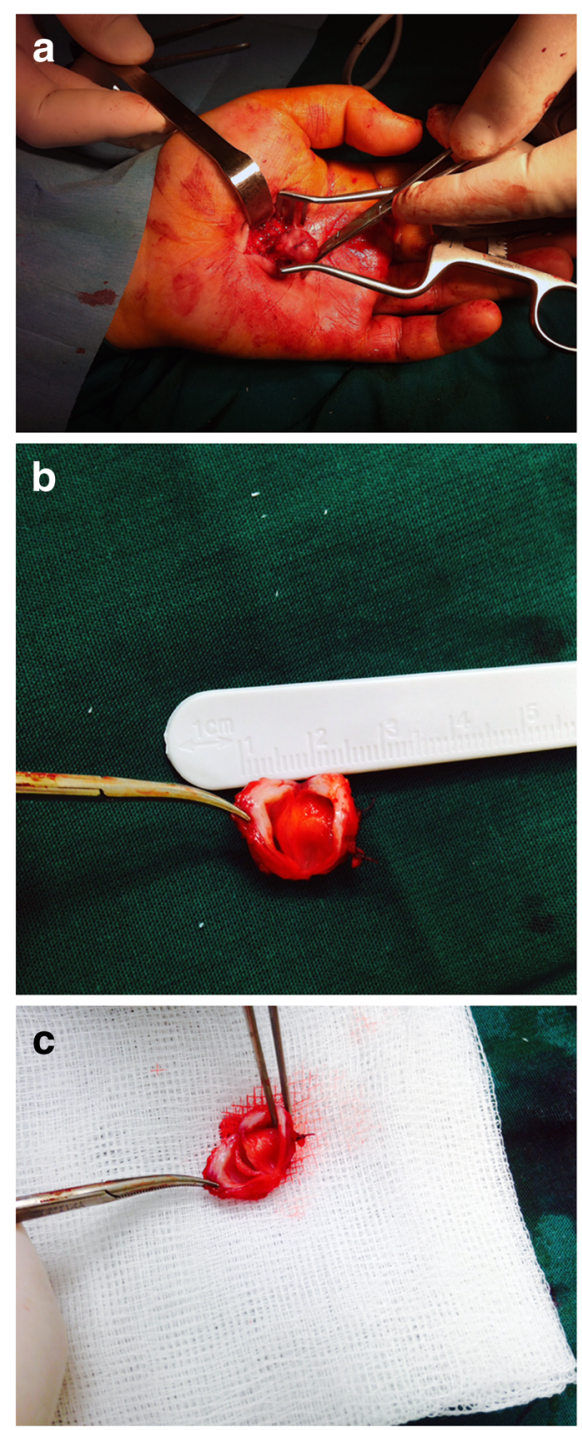

Fig. 3 a, b. Surgical preparation of a $2 \times 2.5 \mathrm{~cm}$ pseudoaneurysm. c. The ligation of the pseudoaneurysm's pedicle is seen on the right

hand injury in order to diagnose the false aneurysm and treat it properly [4].

An X-ray of the hand can be performed to exclude the presence of a foreign body. Ultrasonography is the initial diagnosis in the majority of cases. Angiography can be useful as it can specify the exact location of the aneurysm, the presence of collaterals and may be useful in planning reconstructive surgery, but its use remains controversial due to its potential complications, such as the possibility of distal embolization [5]. In addition, according to Garcia et al., angiography can differentiate the true from the false aneurysms according to their angiographic characteristics: saccular appearance for the false aneurysms and fusiform shape for the true ones [3]. In many cases in the literature angiography was used to make the diagnosis $[1,3,4]$. In our case, the angiography was spared since it would not modify our surgical plan. Ultrasonography was the preferred method used to diagnose the 
pseudoaneurysm in our case. Other reports suggest that ultrasound could be equally as effective as angiography in the diagnosis of the pseudoaneurysms without the abovementioned complications [6]. Computed tomographic (CT) angiography can also be used for the diagnosis, whereas magnetic resonance angiography (MRA) is as useful without necessitating any contrast medium. Also, stereoscopic MRA may provide additional information regarding the location of the aneurysm [4].

The treatment of these lesions can be a matter of debate. Although conservative methods for the treatment of small, uncomplicated and fully thrombosed false aneurysms such as observation and compression bandage have been described [7-9], this could be dangerous due to the risk of distal embolization with subsequent occlusion of the run-off arteries. Therefore, we consider surgery the treatment of choice for the superficial palmar arch false aneurysms. The surgical methods include open surgical resection with ligation or arterial reconstruction $[3,4]$. Successful reconstruction with endto-end anastomosis using microsurgical techniques have been described in the literature [3, 4]. Restoration of the blood flow is preferable, especially in children [3]. Recently, endovascular techniques such as coil embolization have been employed with success in the treatment of the false aneurysms of the superficial palmar arch. This minimally invasive method may be an alternative to open surgery in the management of these lesions [5].

\section{Conclusion}

In spite of their rarity, pseudoaneurysms of the palmar arch can cause severe discomfort and complications to patients after penetrating injury to the palm of the hand. Accurate diagnosis is based on the awareness of the disease and correct imaging procedures. For that reason, follow-up seems to be necessary after penetrating hand injury in order to diagnose and treat this rare entity. We do not favor conservative treatment as it is associated with high risk of distal embolization but suggest surgical resection with ligation or arterial reconstruction as the treatment of choice.

\section{Compliance with Ethical Standards}

Conflict of Interest The authors declare that they have no conflict of interest.

Ethical approval All procedures performed in studies involving human participants were in accordance with the ethical standards of the institutional and/or national research committee and with the $1964 \mathrm{Hel}-$ sinki declaration and its later amendments or comparable ethical standards.

Human and animal Studies This article does not contain any studies with human participants or animals performed by any of the authors.

Informed Consent Informed consent was obtained from all individual participants included in the study.

\section{References}

1. Sterett WI (1996) Pseudoaneurysm of the superficial palmar arch. J Bone Joint Am 78:1089-1091

2. Slesarenko YA, Dagun AB, Hurst LC (2007) False aneurysm of the superficial palmar arch causing acute carpal tunnel syndrome. Orthopedics 30:493-494

3. Frank D, Christophe S, Michel P (2004) False aneurysm of the palmar arch in a child. J Pediatr Surg 39:117-119

4. Sakamoto A, Arai K (2009) False aneurysm of the superficial palmar arch in a child: a case report. Cases J 2:7985

5. Gull S, Spence AJ, Loan W (2011) Superficial pamar arch aneurysm after carpal tunnel decompression, a rare complication: a case report. Cases Rep Med 595120

6. Hughes CD, Binette C, Babigan A (2012) Pseudoaneurysm in the hand of a three-year-old boy: a case report. J Emerg Med 42:87-89

7. Fields JM, Chandra S, Au AK, Rotte M, Ku BS (2013) Images in emergency medicine. Child with painful palmar mass. Superficial palmar arch pseudoaneurysm. Ann Emerg Med 62:569

8. Cromheecke M, Straalen AM, Damen A (1997) Traumatic aneurysm of a common digital artery. J Hand Surg 22B:416-418

9. Cozzi DA, Morini F, Casati A et al (2003) Radial artery pseudoaneurysm successfully treated by compression bandage. Arch Dis Child 88:165-166 